\title{
Patterns of care and survival outcomes after treatment for uveal melanoma in the post-coms era (20042013): a surveillance, epidemiology, and end results analysis
}

\author{
Yuan James Rao, MD*, Julia Sein, MD²*, Shahed Badiyan, MD³, Julie K. Schwarz, MD, PhD', Todd DeWees, PhD', \\ Perry Grigsby, MD聿, Prabakar Kumar Rao, MD# \\ 'Department of Radiation Oncology, Washington University School of Medicine, St. Lovis, MO, ${ }^{2}$ Department of Ophthalmology \\ and Visual Sciences, Washington University School of Medicine, St. Lovis, MO, ${ }^{3}$ Department of Radiation Oncology. University of Maryland, \\ Baltimore, MD, USA \\ *Both authors contributed equally to this work. \\ \#Both authors contributed equally to this work.
}

\begin{abstract}
Purpose: The Collaborative Ocular Melanoma Study (COMS) established modern treatment recommendations for uveal melanoma. We aim to evaluate patterns of care and survival outcomes in the time after COMS.

Material and methods: The retrospective study population includes 2,611 patients in the SEER database treated for uveal melanoma between 2004-2013. Patients stage were T1-4N0M0. Data analyzed included age, clinical stage, tumor size, race, and treatment. Treatments included enucleation (EN) and globe preserving therapy (GPT), which consisted of limited surgical resection or ablation (LSRA), external beam radiation (EBRT), or brachytherapy (BT). Patients treated with radiation may receive radiation therapy alone (RTA) or radiation therapy and supplemental laser therapy (RT+SLT). We evaluated disease specific survival (DSS) and overall survival (OS) using log-rank statistics, and Cox univariate and multivariate analysis.

Results: The median follow-up was 44 months. Treatment strategy was EN in 538 (20.6\%) patients, LSRA in $80(3.1 \%)$, EBRT in 609 (23.3\%), and BT in 1,384 (53.0\%). 1,876 patients received RTA and 117 received RT+SLT. Enucleation was associated with inferior DSS and OS compared to GPT in multivariate analysis (MVA) $(p<0.01)$. Limited surgical resection or ablation and radiation had similar DSS and OS. Brachytherapy and EBRT had similar DSS and OS. Radiation therapy and supplemental laser therapy was associated with improved DSS compared to RTA in UVA $(p=0.03)$, but not MVA. The 5-year DSS for enucleation, RTA, and RT+SLT were $66.7 \%, 87.0 \%$, and $94.7 \%(p<0.01)$, respectively.

Conclusions: Globe preserving treatments such as limited surgery or radiation are commonly utilized alternatives to enucleation, and resulted in favorable survival outcomes. Additional research is required to compare the outcomes of the various globe preserving treatment strategies.

Key words: brachytherapy, enucleation, epidemiology and end results (SEER), laser, transpupillary thermotherapy (TTT), uveal melanoma.

\section{Purpose}

Choroidal melanoma is a rare but life threatening tumor with an incidence of approximately 1,400 new cases in the US every year, with an incidence of approximately 5 per 1 million individuals [1,2]. The Collaborative Ocular Melanoma Study (COMS) demonstrated similar mortality rates when comparing enucleation to plaque radiotherapy for medium sized tumors [3]. As survival rates are similar, selection of treatment has shifted toward eye preserving modalities $[4,5,6]$. Local eye conservation therapy allows for the treatment of tumors with $80 \%$ probability of globe salvage and preservation of some visual function [7].

Plaque radiotherapy has become an important eye conserving treatment for choroidal melanomas. As a form 
of brachytherapy, it provides radiation to the tumor with a surgically implanted radioactive plaque placed on the sclera directly over the intraocular tumor. Proton beam radiation is an alternative to brachytherapy, which delivers a homogenous dose to the tumor, and may have an advantage when the tumor is adjacent to sensitive areas such as the fovea or optic nerve, as it spares normal surrounding tissues. Local control after proton radiation may be comparable to plaque radiotherapy [8]. Other methods of external radiotherapy, such as radiosurgery, have also been employed [9]. Prior retrospective cancer registry studies have suggested that external beam radiation may have similar survival outcomes compared to brachytherapy [10]. However, their comparative effectiveness has not yet been established through prospective randomized studies.

Another form of localized therapy is transpupillary thermotherapy (TTT), which uses infrared radiation to deliver long diode laser treatment through the pupil to the choroidal melanoma, resulting in tumor necrosis [11]. Transpupillary thermotherapy as a sole therapeutic option is limited to a select group of patients with more pigmented tumors, less than $3.5 \mathrm{~mm}$ in thickness, base diameter of less than 10.0, and tumors not abutting the optic nerve [12]. For larger tumors, tumors touching the optic disc, or tumors with high-risk features, thermotherapy may be inadequate as the sole therapy, and plaque radiotherapy or combined therapy may yield a better outcome $[12,13,14]$. Few studies have reported the outcomes of combination of plaque radiotherapy and supplemental TTT in the management of choroidal melanomas $[13,15,16]$.

Lastly, local resection of uveal melanoma can be done through a scleral opening (exoresection) or in a piecemeal fashion with a vitreous cutter (endoresection). Though few surgeons utilize this treatment because of its technical difficulty, local resection has demonstrated conservation of the eye as well as vision [17].

Singh et al. previously reported a trend towards more conservative management in uveal melanoma [1]. However, there is still limited data in the post-COMS era evaluating the patterns of care in the population of patients with uveal melanoma, or comparing survival outcomes of patients treated with enucleation and the various globe preserving treatments. This retrospective study aims to use surveillance, epidemiology, and end results data to broadly evaluate patterns of care, and compare disease specific and all-cause mortality following different treatment modalities including enucleation, brachytherapy, external beam radiation, and local therapy (laser ablation or local resection) in the modern era in the United States.

\section{Material and methods}

\section{Data source and study population}

This analysis was conducted with retrospective data from 18 registries of the National Cancer Institute's Surveillance, Epidemiology and End Results (SEER) program. The SEER 18 registries cover about $30 \%$ of the US population in 13 states. The SEER Program registries collect data on patient demographics, primary tumor site, tumor morphology and stage at diagnosis, first course of treatment, and follow-up for vital status. A retrospective analysis was performed using de-identified data gathered from an individual case query on the SEER database using SEER ${ }^{*}$ tat software version 8.5.3 [18]. The project was exempted by the institutional review board.

Selection criteria included diagnosis year 2004-2013, adult patients age 18-80, first primary cancer, and eye/orbit site. Allowed International Classification of Diseases for Oncology (ICD-O-3) [19] histology codes were 8720, 8730, and 8770-8774 corresponding to melanoma histology. A case file of 3,772 patients was generated from a search using the above criteria. After review, 1,161 patients were excluded from the study due to pre-defined exclusion criteria such as no definitive treatment $(n=88)$, unknown or incomplete staging data $(n=722)$, metastatic disease at diagnosis $(n=57)$, and conjunctiva location $(n=182)$. Non-standard treatment such as combined external beam radiation therapy (EBRT) and brachytherapy (BT) $(n=10)$, enucleation (EN) and radiotherapy $(n=39)$, or photodynamic therapy $(n=4)$ were also excluded. A total of 2,611 remaining eligible patients were included in the study.

Uveal melanomas, as defined in this study, are tumors with ciliary body or posterior choroidal locations differentiated from iris and conjunctiva melanomas. Conjunctival melanomas were not included as they are managed differently and have a different staging system compared to uveal melanomas. Uveal melanoma staging was defined using the American Joint Commission on Cancer (AJCC) $6^{\text {th }}$ edition staging system [20]. Included patients were AJCC $6^{\text {th }}$ edition stage T1-T4, N0, M0.

\section{Statistics}

Four comparisons between treatment groups and techniques were defined to evaluate survival outcomes of commonly used treatment strategies for uveal melanoma as seen in Figure 1. Comparison 1 evaluates enucleation (EN) vs. globe preserving treatment (GPT). Globe preserving treatment was defined as any definitive treatment that spared the eye, and could include limited surgical resection or ablation (LSRA), or radiation therapy (RT). Explicitly, GPT included those patients treated with LSRA, EBRT, or BT. Comparison 2 evaluates LSRA vs. RT. Radiation for uveal melanoma can be given as EBRT or plaque BT. External beam radiation therapy for uveal melanoma is usually either proton radiotherapy or stereotactic radiosurgery. Comparison 3 evaluates the radiation techniques of BT vs. EBRT. Supplementary laser therapy is also given as adjuvant therapy to radiation in some patients. While the laser technique is not coded in SEER in the modern era, TTT is the most common type of laser treatment delivered before or after radiation [13]. Therefore, the terms are used interchangeably for the remainder of the study. Comparison 4 evaluates radiation therapy alone (RTA) without adjuvant laser therapy vs. radiation therapy and supplemental laser therapy (RT+SLT). Ciliary body and choroidal locations as well as all tumor sizes were included in the analysis of the treatment comparisons to evaluate patterns of care, as well as survival. 


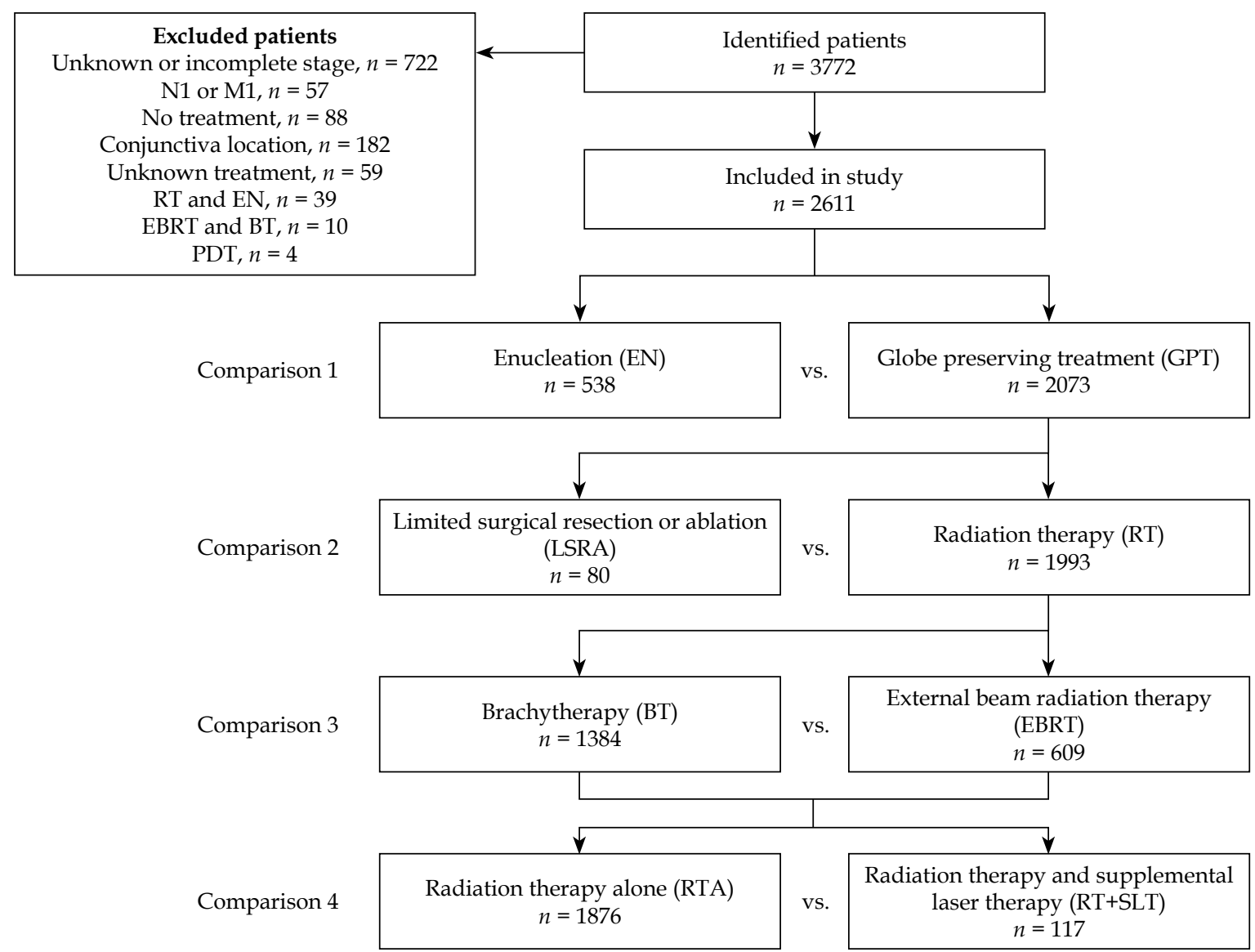

Fig. 1. Flow diagram of study population and treatment comparisons

Disease specific survival (DSS), which is defined as time to death from uveal melanoma, and overall survival (OS), which is defined as time to death from any cause, were analyzed for all comparisons. In SEER, the follow-up time is defined as the time from diagnosis. The Wilcoxon rank sum, Fisher's exact test, and $\chi^{2}$ test were used to evaluate contingency tables. The Kaplan-Meier method was used to estimate survival, and the log-rank test was used to compare survival curves. Univariate Cox proportional hazards models were developed for each treatment comparison and for other covariates. Variables entered into the Cox proportional hazards analysis included the treatment strategy, age, sex, race, year of treatment, T-stage, and tumor location. The analysis was repeated with the addition of tumor size as covariate, as this important factor was known for only a subgroup of patients. Significant factors from the univariate analysis were entered into the multivariate model using a forward stepwise procedure. All statistical tests were two-sided and considered significant at $p<0.05$. Statistical analyses were performed with the Statistical Package for Social Sciences, version 22 (IBM SPSS Statistics, Chicago, IL, USA).

\section{Results}

\section{Patient, tumor, and treatment characteristics}

The study population of 2,611 patients had a median follow-up of 44 months. Mean age at diagnosis was 58 years (range, 18-80 years). Tumor stage was T1 in 987
(37.8\%) patients, T2 in 1,182 (45.3\%), T3 in $401(15.3 \%)$, and T4 in 41 (1.6\%). Tumor location was choroid in 2,303 $(88.2 \%)$ of patients and ciliary body in 308 (11.8\%). Of 1,166 patients with known tumor diameter, 411 (35.2\%) were small size $(\leq 10 \mathrm{~mm}), 478(41.0 \%)$ were medium size (11-16 mm), and 277 (23.8\%) were large size (> $16 \mathrm{~mm})$.

Enucleation was performed on $538(21 \%)$ patients, and GPT was performed on 2,073 (79\%) patients. Limited surgical resection or ablation was the treatment given to $80(3 \%)$ patients, and RT was delivered to 1,993 (76\%) patients. Brachytherapy was delivered to 1,384 (53\%) patients, and EBRT was delivered to 609 (23\%). Among patients receiving radiation, 1,878 (72\%) received RTA without supplemental laser therapy, and $117(4 \%)$ received RT+SLT. The supplemental laser therapy was delivered after radiation in 96 patients, before radiation in 20 patients, and the sequence was unknown in one patient. Additional patient, tumor, and treatment information is documented in Table 1.

\section{Cause of death and survival}

Among 514 patients who died in the follow-up period, the most common causes of death were uveal melanoma in 299 (58.2\%) patients, other malignancy in 95 (18.5\%), and cardiovascular disease in 43 (8.4\%) (Table 2). Among all patients, the 3, 5, and 8-year DSS was $89.6 \%, 84.1 \%$, and $77.3 \%$, respectively; and the 3, 5, and 8-year OS was $86.3 \%, 76.8 \%$, and $67.2 \%$, respectively. The 5-year DSS 
Table 1. Demographics and treatment in the study population

\begin{tabular}{|c|c|c|c|c|c|c|c|c|c|c|}
\hline \multirow[t]{4}{*}{ Factor } & \multicolumn{2}{|c|}{ Enucleation } & \multicolumn{6}{|c|}{ Globe preserving treatment } & \multirow{4}{*}{$\begin{array}{c}\text { EN vs. } \\
\text { GPT } \\
p \text { value }\end{array}$} & \multirow{4}{*}{$\begin{array}{c}\text { LSRA vs. } \\
\text { RT } \\
p \text { value }\end{array}$} \\
\hline & \multirow[t]{3}{*}{$n$} & \multirow[t]{3}{*}{$\%$} & \multicolumn{2}{|c|}{ LSRA } & \multicolumn{4}{|c|}{ Radiation } & & \\
\hline & & & \multirow[t]{2}{*}{$n$} & \multirow[t]{2}{*}{$\%$} & \multicolumn{2}{|c|}{ EBRT } & \multicolumn{2}{|c|}{ BT } & & \\
\hline & & & & & $n$ & $\%$ & $n$ & $\%$ & & \\
\hline Total patients & 538 & & 80 & & 609 & & 1384 & & & \\
\hline Follow-up (median) & \multicolumn{2}{|c|}{36 months } & \multicolumn{2}{|c|}{65 months } & \multicolumn{2}{|c|}{50 months } & \multicolumn{2}{|c|}{44 months } & $<0.01$ & $<0.01$ \\
\hline Age (mean) & \multicolumn{2}{|c|}{58 years } & \multicolumn{2}{|c|}{56 years } & \multicolumn{2}{|c|}{59 years } & \multicolumn{2}{|c|}{59 years } & ns & ns \\
\hline$\leq 60$ years & 284 & 52.8 & 45 & 56.3 & 333 & 54.7 & 757 & 54.7 & & \\
\hline$>60$ years & 254 & 47.2 & 35 & 43.8 & 276 & 45.3 & 627 & 45.3 & ns & ns \\
\hline \multicolumn{11}{|l|}{ Sex } \\
\hline Male & 320 & 59.5 & 42 & 52.5 & 305 & 50.1 & 727 & 52.5 & & \\
\hline Female & 218 & 40.5 & 38 & 47.5 & 304 & 49.9 & 657 & 47.5 & $<0.01$ & ns \\
\hline \multicolumn{11}{|l|}{ Race } \\
\hline White & 516 & 95.9 & 77 & 96.3 & 581 & 95.4 & 1321 & 95.4 & & \\
\hline Other & 22 & 4.1 & 3 & 3.8 & 28 & 4.6 & 63 & 4.6 & ns & ns \\
\hline \multicolumn{11}{|l|}{ Treatment year } \\
\hline 2004-2008 & 293 & 54.5 & 51 & 63.8 & 312 & 51.2 & 618 & 44.7 & & \\
\hline 2009-2013 & 245 & 45.5 & 29 & 36.3 & 297 & 48.8 & 766 & 55.3 & $<0.01$ & $<0.01$ \\
\hline T-stage & & & & & & & & & & \\
\hline $\mathrm{T} 1$ & 165 & 30.7 & 64 & 80.0 & 182 & 29.9 & 576 & 41.6 & & \\
\hline $\mathrm{T} 2$ & 179 & 33.3 & 12 & 15.0 & 315 & 51.7 & 676 & 48.8 & & \\
\hline T3 & 174 & 32.3 & 4 & 5.0 & 103 & 16.9 & 120 & 8.7 & & \\
\hline T4 & 20 & 3.7 & 0 & 0.0 & 9 & 1.5 & 12 & 0.9 & $<0.01$ & $<0.01$ \\
\hline Tumor diameter & & & & & & & & & & \\
\hline Unknown & 287 & - & 56 & - & 280 & - & 822 & - & & \\
\hline Small $(\leq 10 \mathrm{~mm})$ & 70 & 27.9 & 15 & 62.5 & 124 & 37.7 & 202 & 35.9 & & \\
\hline Medium (11-16 mm) & 103 & 41.0 & 2 & 8.3 & 124 & 37.7 & 249 & 44.3 & & \\
\hline Large (> $16 \mathrm{~mm})$ & 78 & 31.1 & 7 & 29.2 & 81 & 24.6 & 111 & 19.8 & $<0.01$ & $<0.01$ \\
\hline Tumor site & & & & & & & & & & \\
\hline Choroid & 454 & 84.4 & 20 & 25.0 & 535 & 87.8 & 1294 & 93.4 & & \\
\hline Ciliary body & 84 & 15.6 & 60 & 75.0 & 74 & 12.2 & 90 & 6.5 & $<0.01$ & $<0.01$ \\
\hline Laser therapy & & & & & & & & & & \\
\hline No & 539 & 100.2 & 67 & 83.8 & 595 & 97.7 & 1281 & 92.6 & & \\
\hline Yes & 0 & 0.0 & 13 & 16.3 & 14 & 2.3 & 103 & 7.4 & $<0.01$ & $<0.01$ \\
\hline Surgery received & & & & & & & & & & \\
\hline Plaque brachytherapy & 0 & 0.0 & 0 & 0.0 & 0 & 0.0 & 1385 & 100.0 & & \\
\hline Local tumor ablation & 0 & 0.0 & 14 & 17.5 & 17 & 54.8 & 113 & 86.3 & & \\
\hline Local tumor excision & 0 & 0.0 & 66 & 82.5 & 17 & 54.8 & 18 & 13.7 & & \\
\hline Enucleation & 538 & 100.0 & 0 & 0.0 & 0 & 0.0 & 0 & 0.0 & $<0.01$ & $<0.01$ \\
\hline
\end{tabular}

LSRA - limited surgical resection or ablation, EBRT - external beam radiation, BT - brachytherapy, EN - enucleation, GPT - globe preserving therapy

for stage $\mathrm{T} 1, \mathrm{~T} 2, \mathrm{~T} 3$, and $\mathrm{T} 4$ tumors were $90.6 \%, 82.0 \%$, $71.9 \%$, and $68.3 \%$ respectively $(p<0.01)$. The 5 -year DSS for small, medium, and large size tumors were $94.3 \%$, $76.2 \%$, and $78.6 \%$, respectively $(p<0.01)$. The 5 -year DSS for EN, LSRA, RTA, and RT+SLT were $66.7 \%, 94.8 \%$, $87.0 \%$, and $94.7 \%$, respectively $(p<0.01)$. Disease specific survival and OS outcomes according to treatment,
T-stage, tumor diameter, and tumor location are shown in Figures 2-4.

Age $>60$, white race, higher T-stage, and larger tumor diameter were associated with increased risk of death from uveal melanoma on univariate analysis as seen in Table 3. These significant factors were entered into a multivariate model for evaluation of the treatment comparisons as seen 
in Table 3. The multivariate model adjusted for the extent of the tumor using the T-stage, which incorporates the size and thickness of the tumor, but the model did not utilize the exact tumor diameter, as this measurement was unknown for 1,445 (55\%) patients. A second multivariate model on the subset of patients with known tumor size was generated incorporating exact tumor diameter as a continuous variable shown in Table 4. Post-hoc subgroup analyses based on T-stage, size, and location for each treatment comparison for DSS are also presented in Table 5. The following comparisons of treatment strategies incorporate the interpretation of patient outcomes from both multivariate models and the subset analysis, but hazard ratios are reported from the first model as it incorporates the greatest number of patients.

\section{Comparison 1: Enucleation vs. globe preserving therapy}

Comparison 1 included all 2,611 patients, and directly compared survival for 538 patients treated with enucleation vs. 2,073 patients treated with globe preserving therapy. These two groups were similarly balanced by age and race. Patients receiving enucleation were more likely to be diagnosed prior to year $2008(p<0.01)$, male $(p<0.01)$, higher T stage $(p<0.01)$, larger tumor size

A Treatment

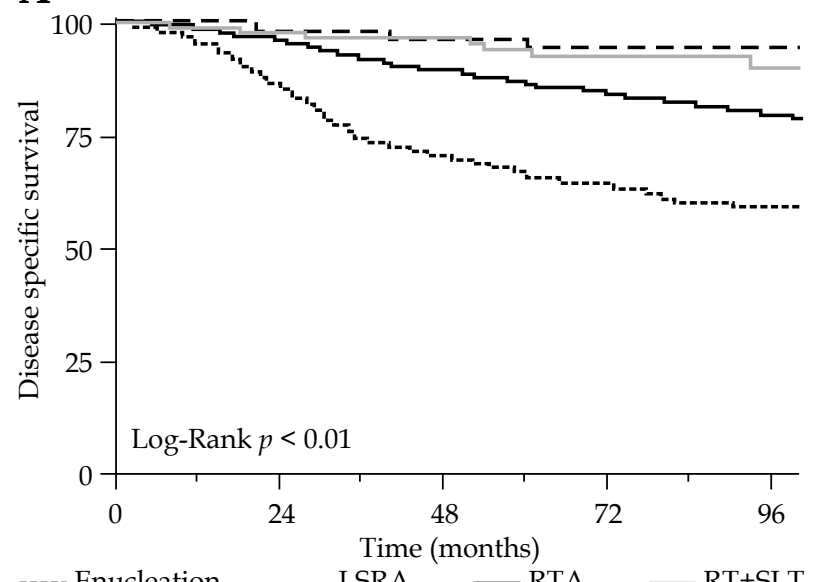

Table 2. Causes of death in the study population

\begin{tabular}{lcc} 
Cause of death & Frequency & $\%$ \\
\hline Uveal melanoma & 299 & 58.2 \\
\hline Other cancer & 95 & 18.5 \\
\hline Cardiovascular disease & 43 & 8.4 \\
\hline Cerebrovascular disease & 9 & 1.8 \\
\hline Infection & 9 & 1.8 \\
\hline Accident & 7 & 1.4 \\
\hline Chronic obstructive pulmonary disease & 6 & 1.2 \\
\hline Kidney disease & 4 & 0.8 \\
\hline Liver disease & 3 & 0.6 \\
\hline Diabetes & 3 & 0.6 \\
\hline Alzheimer's disease & 2 & 0.4 \\
\hline Suicide & 2 & 0.4 \\
\hline Unknown cause of death & 32 & 6.2 \\
\hline Total & 514 & 100.0
\end{tabular}
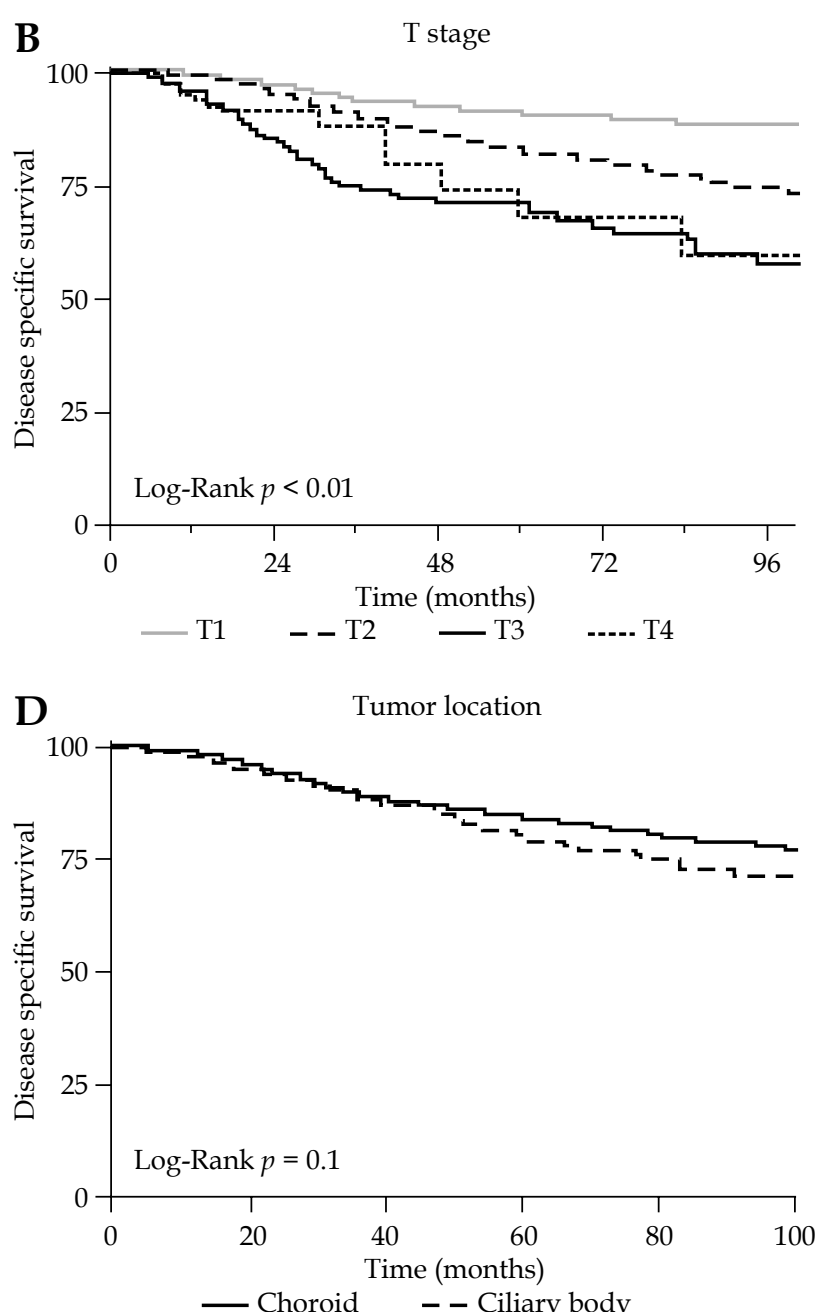

C

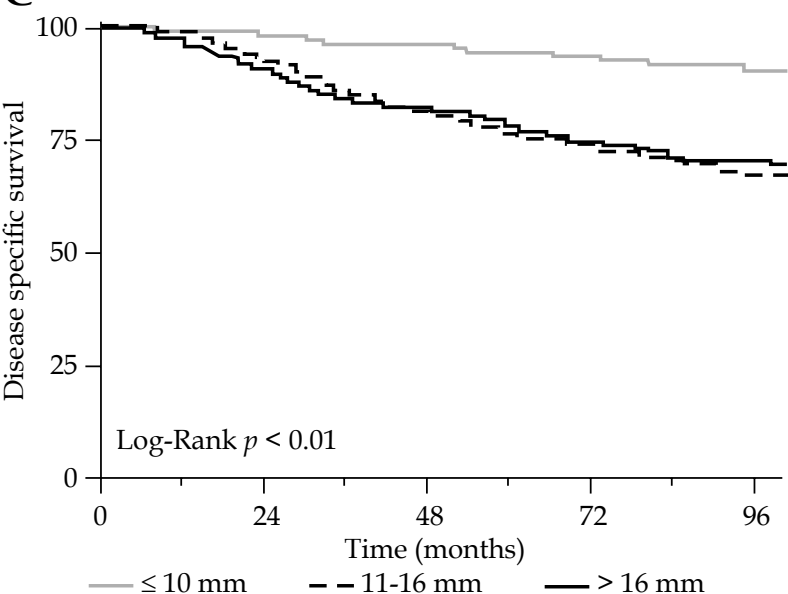

Fig. 2. Disease specific survival according to treatment, t-stage, tumor diameter, and tumor location 
A

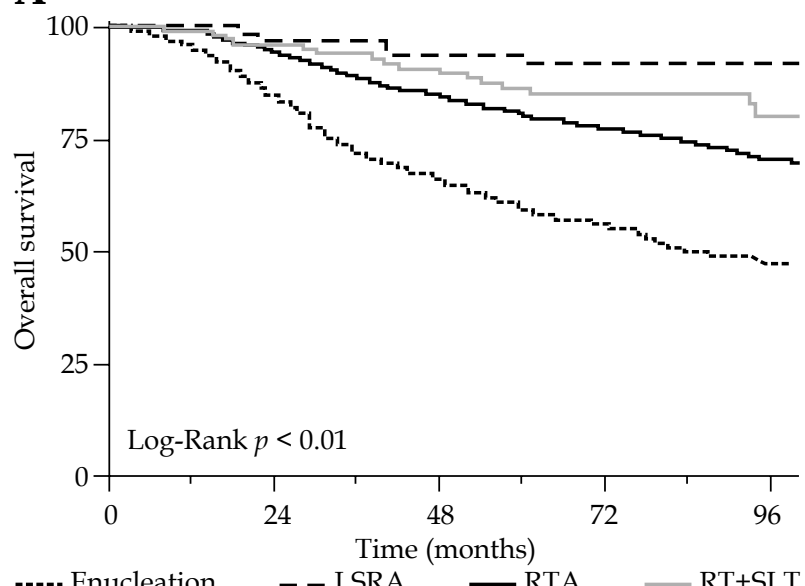

..... Enucleation - - LSRA - RTA RT+SLT

C

Tumor diameter

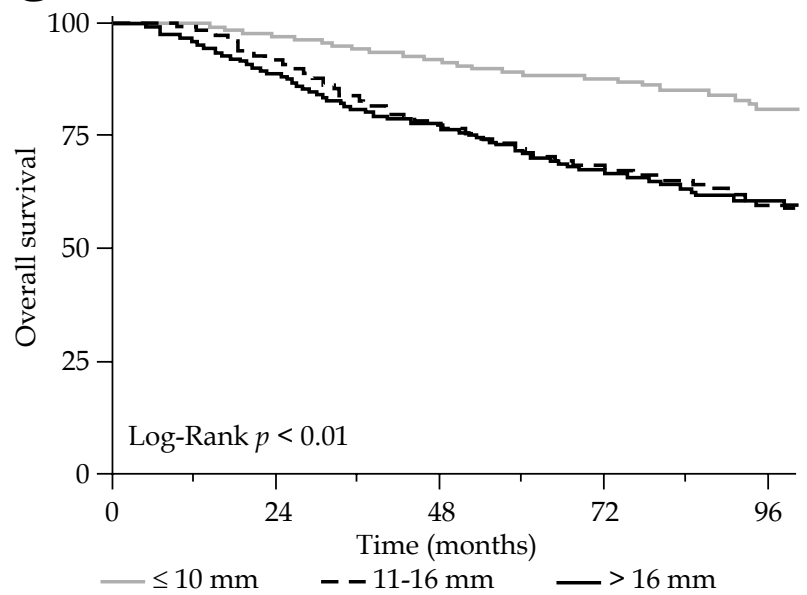

B

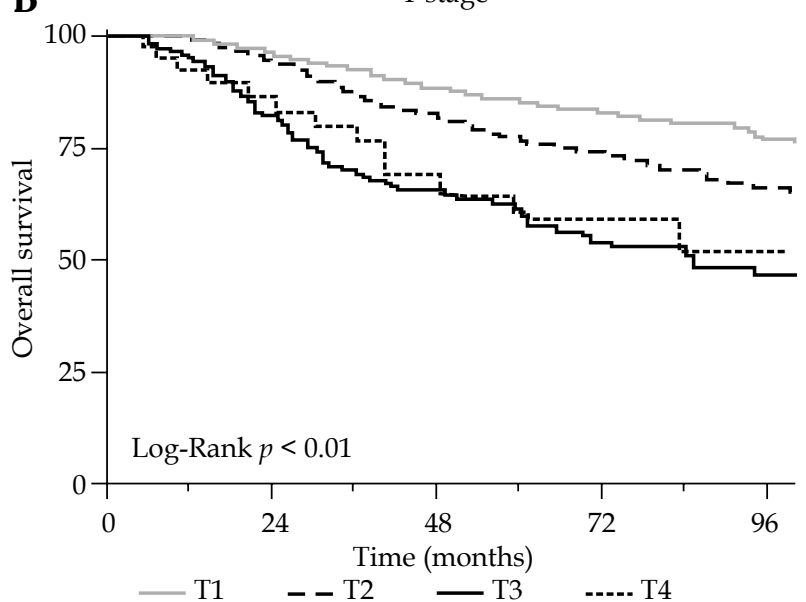

D

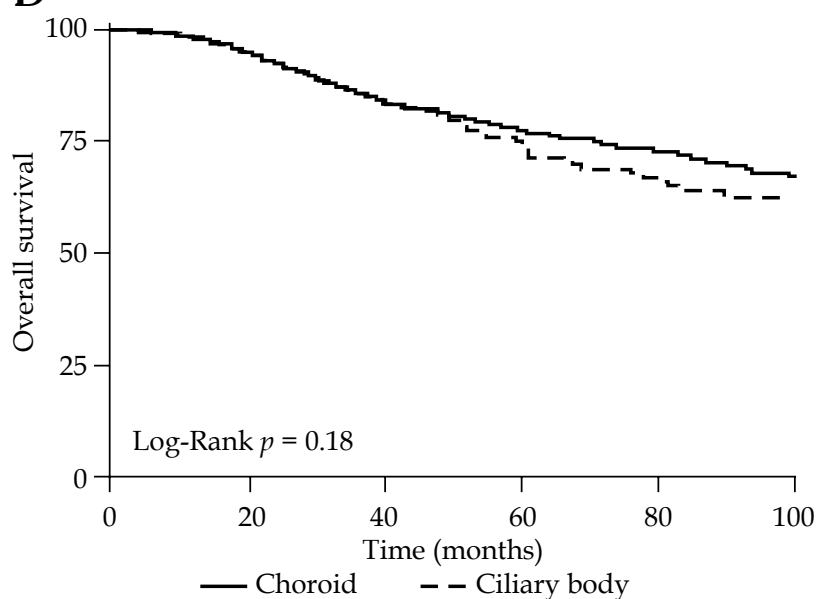

Fig. 3. Overall survival according to treatment, t-stage, tumor diameter, and tumor location

$(p<0.01)$, and ciliary body location $(p<0.01)$. Five-year DSS for enucleation vs. globe preserving treatment were $66.7 \%$ and $87.9 \%(p<0.01)$ (Figure $5 \mathrm{~A})$. The 5-year OS for EN vs. GPT were $59.2 \%$ and $81.4 \%(p<0.01)$ (Figure $4 \mathrm{~A}$ ). Globe preserving therapy was associated with superior DSS and OS on univariate and multivariate analysis compared to EN (multivariate GPT vs. EN DSS HR 0.36, 95\% CI: $0.29-0.45, p<0.01)$, when adjusted for age, race, and T-stage as seen in Table 3. The associations remained significant when additionally adjusted for exact tumor size as seen in Table 4, and are observed in every subgroup as presented in Table 5 .

\section{Comparison 2: Local surgical resection or ablation vs. radiation therapy}

Comparison 2 included the 2,073 patients receiving GPT, and directly compared survival for 80 patients receiving LSRA vs. 1,993 patients receiving RT. Of the patients receiving LSRA, 14 received local tumor ablation and 66 received local tumor excision. The two patient groups were similarly balanced by age, sex, and race. Patients receiving LSRA were more likely to be diagnosed prior to year $2008(p<0.01)$, early stage $(p<0.01)$, small tumor $(p<0.01)$, and located in ciliary body $(p<0.01)$.
Five-year DSS for LSRA vs. RT was $94.8 \%$ and $87.6 \%$ $(p=0.02)$ (Figure 5B). The 5-year OS for LSRA vs. GPT were $91.8 \%$ and $80.9 \%(p<0.01)$ (Figure 4B). Local surgical resection or ablation was associated with superior DSS and OS on univariate analysis compared to RT (univariate RT vs. LSRA DSS HR 3.71, 95\% CI: 1.18-11.59, $p=0.02$ ). However, this comparison was not significant for DSS or OS on multivariate analysis. In subgroup analysis, LSRA may be associated with improved DSS compared to RT in patients with ciliary body tumors as seen in Table 5 .

\section{Comparison 3: External beam radiation therapy vs. brachytherapy}

Comparison 3 included the 1,993 patients receiving $\mathrm{RT}$, and directly compared survival for 1,384 patients receiving BT vs. 609 patients receiving EBRT. These two groups were similarly balanced by age and race. External beam radiation therapy was more likely to be used in patients treated before year $2008(p<0.01)$, higher T-stage $(p<0.01)$, and larger tumors $(p<0.01)$. Five-year DSS for EBRT vs. BT were $86.7 \%$ and $88.0 \%(p=0.45)$ (Figure 5 C). The 5-year OS for EBRT vs. BT were $80.5 \%$ and $81.1 \%$ $(p=0.77$ ) (Figure 4C). There was no significant difference in DSS or OS between patients treated with EBRT vs. BT 
A

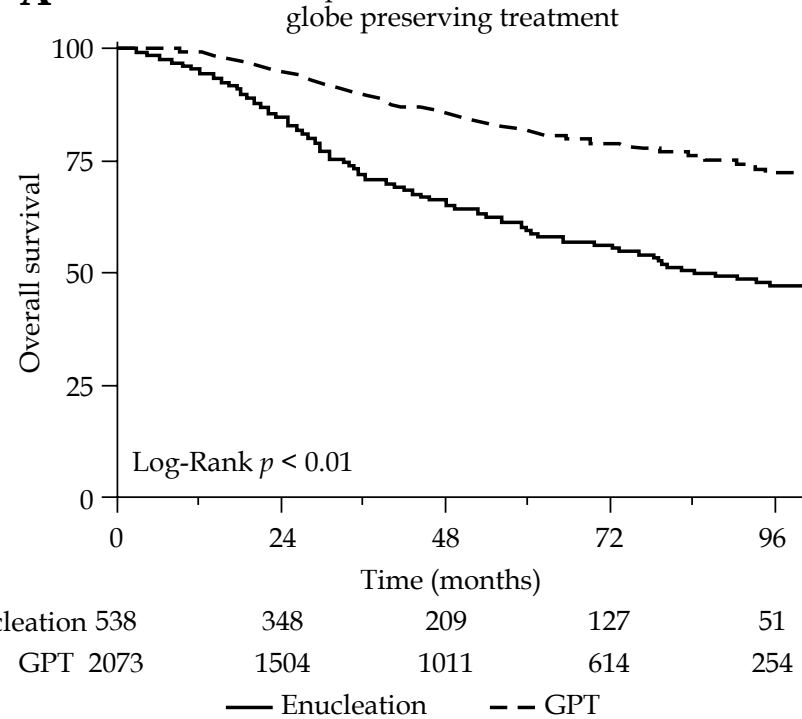

C Comparison 3: External beam radiation therapy vs.

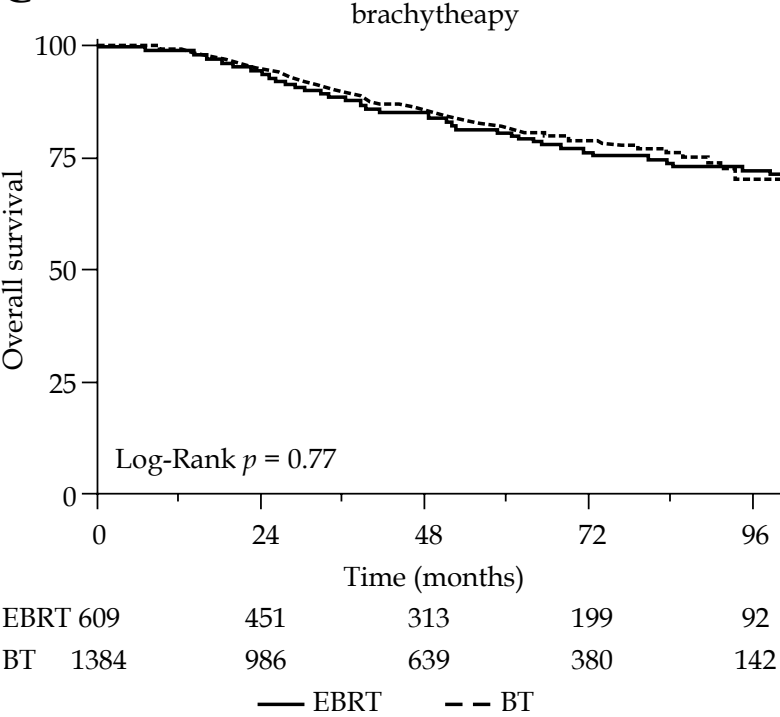

Fig. 4. Overall survival according to treatment comparisons

on univariate and multivariate analysis or in subgroup analyses.

\section{Comparison 4: Radiation therapy alone vs.} radiation therapy and supplemental laser therapy

Comparison 4 included the 1,993 patients receiving radiation therapy, and directly compared survival for 1,876 patients receiving radiation therapy alone vs. 117 patients receiving radiation therapy and supplemental laser therapy. Age, sex, and race was balanced between groups. Radiation therapy and supplemental laser therapy was more likely to be used prior to year $2008(p<0.01)$, lower T-stage $(p<0.01)$, smaller size $(p<0.01)$, and choroid location $(p=0.02)$. Five-year DSS for RTA vs. RTSLT was $87.0 \%$ vs. $94.7 \%(p=0.03)$ (Figure $5 \mathrm{D})$. The 5 -year OS for RTA vs. RTSLT were $80.5 \%$ and $86.5 \%(p=0.06)$ (Figure 4C). Radiation therapy and supplemental laser therapy was associated with improved DSS compared to
B Comparison 2: Local surgical resection or ablation vs.

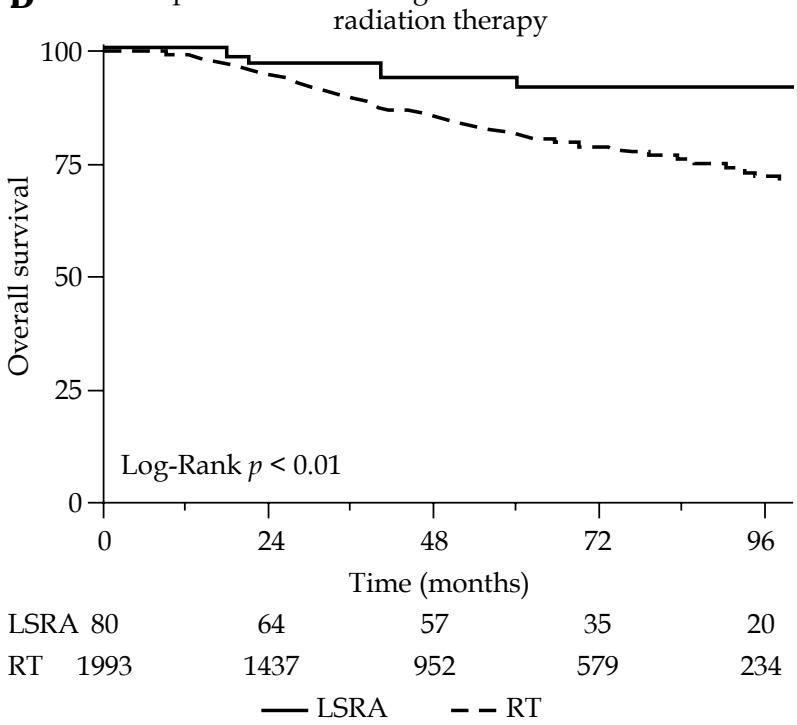

D Comparison 4: Radiation therapy alone vs. radiation therapy and supplemental laser therapy

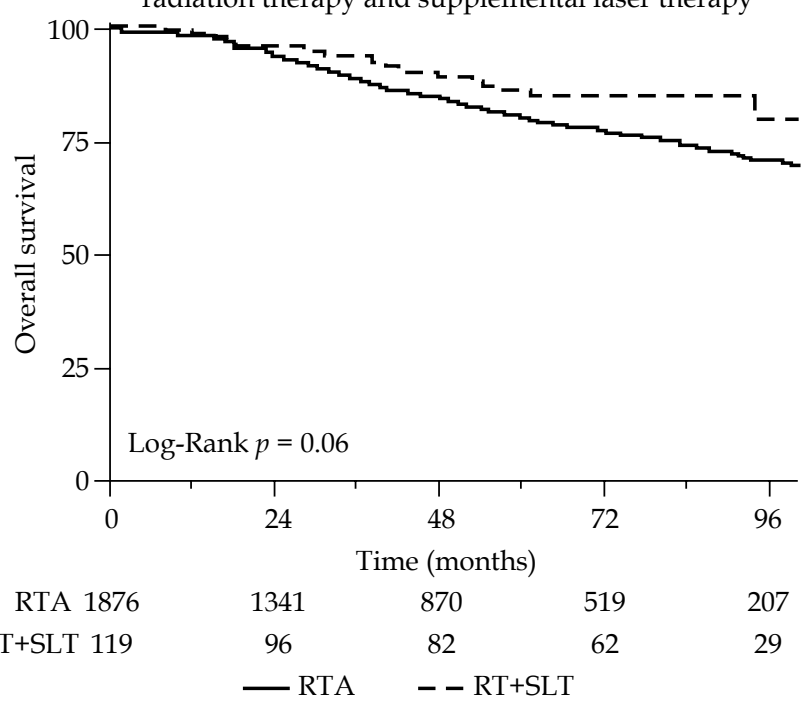

RTA (univariate RTSLT vs. RTA DSS HR 0.46, 95\% CI: $0.22-0.94, p=0.03$ ) in the univariate analysis. However, this benefit was not observed in the multivariate analysis. In subgroup analysis, the benefit of supplemental laser therapy appears to be in patients with T1 or T2 tumors, and tumors in choroid locations as presented in Table 5. There was no significant effect on the sequence of laser treatment and radiation on DSS $(p=0.08)$ or OS $(p=0.50)$.

\section{Discussion}

\section{Collaborative Ocular Melanoma Study and indications for enucleation}

The Collaborative Ocular Melanoma Study concluded that there was no difference in the 5-year and 12-year melanoma-related mortality with medium sized choroidal melanomas treated with ${ }^{125} \mathrm{I}$ brachytherapy or enucle- 
Table 3. Univariate and multivariate analysis of treatment comparisons and covariates for disease specific and overall survival

\begin{tabular}{|c|c|c|c|c|c|c|c|c|}
\hline \multirow[t]{2}{*}{ Factor } & \multicolumn{2}{|c|}{ UVA of DSS } & \multicolumn{2}{|c|}{ MVA of DSS } & \multicolumn{2}{|l|}{ UVA of OS } & \multicolumn{2}{|c|}{ MVA of OS } \\
\hline & $\mathrm{HR}(95 \% \mathrm{Cl})$ & $p$ & HR $(95 \% \mathrm{Cl})$ & $p$ & HR $(95 \% \mathrm{Cl})$ & $p$ & $\mathrm{HR}(95 \% \mathrm{Cl})$ & $p$ \\
\hline \multicolumn{9}{|l|}{ Comparison 1} \\
\hline EN & Reference & & Reference & & Reference & & Reference & \\
\hline GPT & $0.32(0.26-0.40)$ & $<0.01$ & $0.36(0.29-0.45)$ & $<0.01$ & $0.40(0.33-0.47)$ & $<0.01$ & $0.45(0.37-0.54)$ & $<0.01$ \\
\hline \multicolumn{9}{|l|}{ Comparison 2} \\
\hline LSRA & Reference & & & & Reference & & & \\
\hline RT & $3.71(1.18-11.59)$ & 0.02 & & 0.17 & $3.57(1.48-8.63)$ & $<0.01$ & & 0.05 \\
\hline \multicolumn{9}{|l|}{ Comparison 3} \\
\hline EBRT & Reference & & & & Reference & & & \\
\hline BT & $0.88(0.66-1.18)$ & 0.39 & & 0.20 & $0.96(0.76-1.20)$ & 0.72 & & 0.38 \\
\hline \multicolumn{9}{|l|}{ Comparison 4} \\
\hline RTA & Reference & & & & Reference & & & \\
\hline $\mathrm{RT}+\mathrm{SLT}$ & $0.46(0.22-0.94)$ & 0.03 & & 0.18 & $0.62(0.38-1.01)$ & 0.06 & & 0.21 \\
\hline \multicolumn{9}{|l|}{ Age } \\
\hline$\leq 60$ years & Reference & & Reference & & Reference & & Reference & \\
\hline$>60$ years & $1.56(1.26-1.92)$ & $<0.01$ & $1.59(1.28-1.95)$ & $<0.01$ & $1.999(1.67-2.37)$ & $<0.01$ & $2.00(1.68-2.39)$ & $<0.01$ \\
\hline \multicolumn{9}{|l|}{ Sex } \\
\hline Male & Reference & & & & Reference & & & \\
\hline Female & $0.86(0.69-1.06)$ & 0.16 & & & $0.83(0.70-0.99)$ & 0.04 & & \\
\hline \multicolumn{9}{|l|}{ Race } \\
\hline White & Reference & & Reference & & Reference & & & \\
\hline Non-white & $0.44(0.21-0.93)$ & 0.03 & $0.45(0.21-0.96)$ & 0.04 & $0.73(0.45-1.19)$ & 0.21 & & \\
\hline \multicolumn{9}{|l|}{ Treatment year } \\
\hline $2004-2008$ & Reference & & & & Reference & & & \\
\hline 2009-2013 & $0.96(0.74-1.24)$ & 0.75 & & & $1.03(0.83-1.27)$ & 0.81 & & \\
\hline \multicolumn{9}{|l|}{ T stage } \\
\hline $\mathrm{T} 1$ & Reference & & Reference & & Reference & & Reference & \\
\hline $\mathrm{T} 2$ & $2.15(1.62-2.84)$ & $<0.01$ & $2.26(1.71-2.98)$ & $<0.01$ & $1.64(1.32-2.03)$ & $<0.01$ & $1.70(1.37-2.11)$ & $<0.01$ \\
\hline T3 & $4.54(3.31-6.24)$ & $<0.01$ & $3.61(2.62-4.99)$ & $<0.01$ & $3.48(2.71-4.47)$ & $<0.01$ & $2.95(2.29-3.81)$ & $<0.01$ \\
\hline $\mathrm{T4}$ & $3.56(1.78-7.15)$ & $<0.01$ & $2.72(1.35-5.47)$ & $<0.01$ & $2.84(1.61-5.04)$ & $<0.01$ & $2.34(1.31-4.15)$ & $<0.01$ \\
\hline \multicolumn{9}{|l|}{ Tumor diameter } \\
\hline Small $(\leq 10 \mathrm{~mm})$ & Reference & & & & Reference & & & \\
\hline Medium (11-16 mm) & $4.54(3.03-6.79)$ & $<0.01$ & & & $2.69(2.01-3.60)$ & $<0.01$ & & \\
\hline Large (> $16 \mathrm{~mm})$ & $4.19(2.72-6.45)$ & $<0.01$ & & & $2.84(1.96-3.69)$ & $<0.01$ & & \\
\hline \multicolumn{9}{|l|}{ Tumor site } \\
\hline Choroid & Reference & & & & Reference & & & \\
\hline Ciliary body & $1.27(0.95-1.70)$ & 0.11 & & & $1.18(0.92-1.50)$ & 0.11 & & \\
\hline
\end{tabular}

EN - enucleation, GPT - globe preserving therapy, LSRA - limited surgical resection or ablation, RT - radiation therapy, EBRT - external beam radiation, $B T$ - brachytherapy, RTA - radiation therapy alone, RT+SLT - radiation therapy and laser

ation [21]. Although our analysis appears to suggest that conservative localized therapy may be associated with greater disease specific and overall survival compared to enucleation, even when adjusted for tumor stage and tumor size, this result should be interpreted very cautiously as SEER data is retrospective and non-randomized. Our retrospective data represents a lower level of evidence compared to the previously published COMS randomized studies and does not supersede their conclusions.

Instead, our data does suggest that in the modern era, there has been a shift in the patterns of care towards the use of enucleation in patients with larger tumors or more advanced T-stage, with globe-preserving therapies preferred for those patients with smaller tumors. 
Table 4. Multivariate analysis of treatment comparisons and covariates for disease specific and overall survival, incorporating tumor diameter as a continuous variable

\begin{tabular}{|c|c|c|c|c|}
\hline \multirow[t]{2}{*}{ Factor } & \multicolumn{2}{|c|}{ MVA of DSS } & \multicolumn{2}{|c|}{ MVA of OS } \\
\hline & $\mathrm{HR}(95 \% \mathrm{Cl})$ & $p$ & $\mathrm{HR}(95 \% \mathrm{Cl})$ & $p$ \\
\hline \multicolumn{5}{|l|}{ Comparison 1} \\
\hline$\overline{E N}$ & Reference & \multirow{2}{*}{$<0.01$} & Reference & \multirow{2}{*}{$<0.01$} \\
\hline GPT & $0.35(0.27-0.47)$ & & $0.42(0.33-0.53)$ & \\
\hline \multicolumn{5}{|l|}{ Comparison 2} \\
\hline LSRA & & \multirow{2}{*}{0.58} & & \multirow{2}{*}{0.66} \\
\hline RT & & & & \\
\hline \multicolumn{5}{|l|}{ Comparison 3} \\
\hline EBRT & & \multirow{2}{*}{0.17} & & \multirow{2}{*}{0.12} \\
\hline$\overline{B T}$ & & & & \\
\hline \multicolumn{5}{|l|}{ Comparison 4} \\
\hline RTA & & \multirow{2}{*}{0.36} & & \multirow{2}{*}{0.88} \\
\hline$R T+S L T$ & & & & \\
\hline \multicolumn{5}{|l|}{ Age } \\
\hline$\leq 60$ years & Reference & \multirow{2}{*}{$<0.01$} & Reference & \multirow{2}{*}{$<0.01$} \\
\hline$>60$ years & $1.48(1.14-1.92)$ & & $2.00(1.68-2.39)$ & \\
\hline \multicolumn{5}{|l|}{ Race } \\
\hline White & Reference & \multirow{2}{*}{0.01} & Reference & \multirow{2}{*}{0.03} \\
\hline Non-white & $0.17(0.04-0.69)$ & & $0.43(0.20-0.91)$ & \\
\hline \multicolumn{5}{|l|}{ Tstage } \\
\hline $\mathrm{T} 1$ & Reference & & Reference & \\
\hline $\mathrm{T} 2$ & $3.13(2.10-4.67)$ & $<0.01$ & $2.12(1.58-2.86)$ & $<0.01$ \\
\hline $\mathrm{T} 3$ & $4.28(2.68-6.83)$ & $<0.01$ & $3.16(2.21-4.53)$ & $<0.01$ \\
\hline$\overline{\mathrm{T} 4}$ & $4.45(1.84-10.76)$ & 0.01 & $3.15(1.50-6.63)$ & $<0.01$ \\
\hline Tumor diameter (continuous) & $0.999(0.996-1.002)$ & 0.64 & $1.000(0.997-1.003)$ & 0.90 \\
\hline
\end{tabular}

MVA - multivariate analysis, DSS - disease specific, OS - overall survival, EN - enucleation, GPT - globe preserving therapy, LSRA - limited surgical resection or ablation, $R T$ - radiation therapy, EBRT - external beam radiation, $B T$ - brachytherapy, $R T A$ - radiation therapy alone, $R T+S L T$ - radiation therapy and laser

Table 5. Post-hoc subgroup analysis of treatment comparisons according to tumor stage, tumor size, and tumor site

\begin{tabular}{|c|c|c|c|c|}
\hline \multirow[t]{3}{*}{ Factor } & Comparison 1 & Comparison 2 & Comparison 3 & Comparison 4 \\
\hline & EN vs. GPT & LSRA vs. RT & EBRT vs. BT & RTA vs. RT+SLT \\
\hline & $\mathrm{HR}(95 \% \mathrm{Cl})^{1}$ & $\mathrm{HR}(95 \% \mathrm{Cl})^{2}$ & $\mathrm{HR}(95 \% \mathrm{Cl})^{3}$ & HR $(95 \% \mathrm{Cl})^{4}$ \\
\hline \multicolumn{5}{|l|}{ Tumor stage } \\
\hline $\mathrm{T} 1$ and $\mathrm{T} 2$ & $0.33(0.26-0.43)$ & $3.06(0.98-9.60)$ & $1.00(0.71-1.40)$ & $0.47(0.22-1.00)$ \\
\hline $\mathrm{T} 3$ and $\mathrm{T} 4$ & $0.50(0.33-0.74)$ & 20.65 (0.00-NR) & $0.96(0.53-1.74)$ & $1.14(0.16-8.43)$ \\
\hline \multicolumn{5}{|l|}{ Tumor diameter } \\
\hline Small $(\leq 10 \mathrm{~mm})$ & $0.36(0.17-0.77)$ & $0.92(0.12-6.88)$ & $1.20(0.45-3.21)$ & $0.04(0.00-12.35)$ \\
\hline Medium (11-16 mm) & $0.47(0.32-0.67)$ & $0.36(0.05-2.58)$ & $0.99(0.63-1.55)$ & $0.76(0.33-1.75)$ \\
\hline Large (> $16 \mathrm{~mm})$ & $0.25(0.15-0.39)$ & 21.22 (0.00-NR) & $1.12(0.56-2.24)$ & $0.83(0.11-6.10)$ \\
\hline \multicolumn{5}{|l|}{ Tumor site } \\
\hline Choroid & $0.33(0.27-0.42)$ & $0.98(0.24-3.94)$ & $0.98(0.72-1.35)$ & $0.44(0.21-0.93)$ \\
\hline Ciliary body & $0.27(0.16-0.45)$ & $12.70(1.72-93.9)$ & $0.60(0.27-1.35)$ & $1.32(0.18-9.78)$ \\
\hline
\end{tabular}

EN-enucleation, GPT-globe preserving therapy, LSRA - limited surgical resection or ablation, RT-radiation therapy, EBRT-external beam radiation, BT - brachytherapy, $R T A$ - radiation therapy alone, $R T+S L T$ - radiation therapy and laser

Hazard ratios presented are unadjusted hazard ratios for disease specific survival in the subgroup. NR-not reported, as upper limit of hazard ratio is greater than 100 ${ }^{1}$ Lower number favors globe preserving treatment, ${ }^{2}$ Lower number favors radiation, ${ }^{3}$ Lower number favors brachytherapy, ${ }^{4}$ Lower number favors radiation and supplemental laser treatment 
A
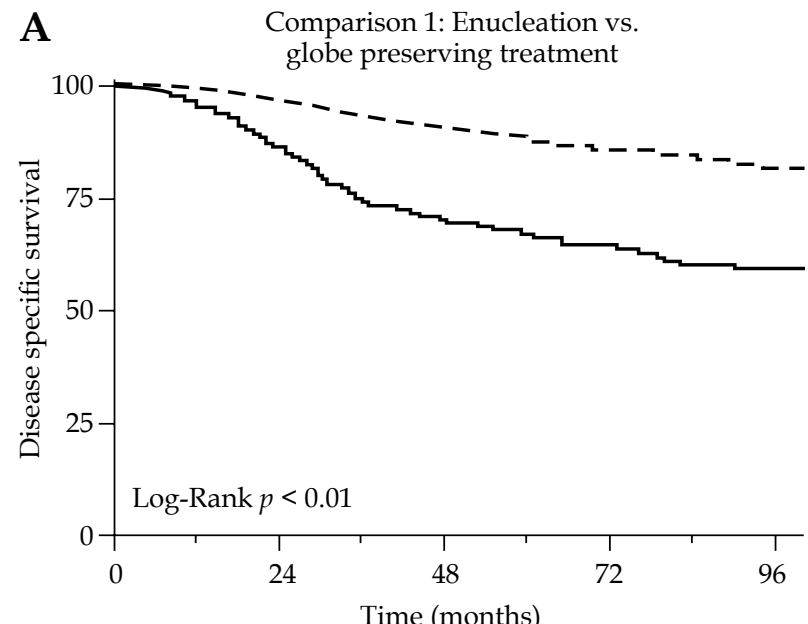

Enucleation 538

GPT 2073

$\begin{array}{lrr}348 & 209 & 127 \\ 1504 & 1011 & 614 \\ - \text { Enucleation } & - & \text { - GPT }\end{array}$

C Comparison 3: External beam radiation therapy vs. brachytheapy

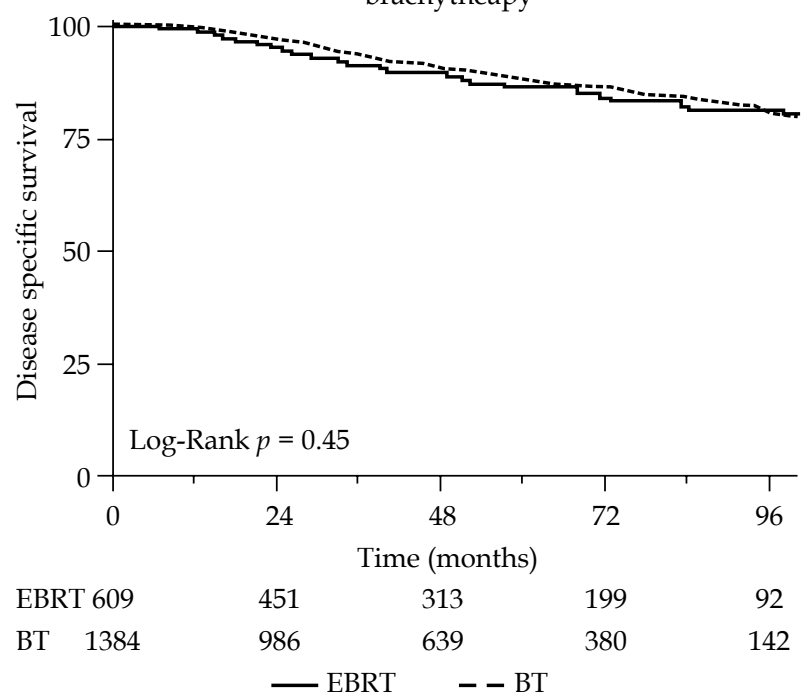

B Comparison 2: Local surgical resection or ablation vs.

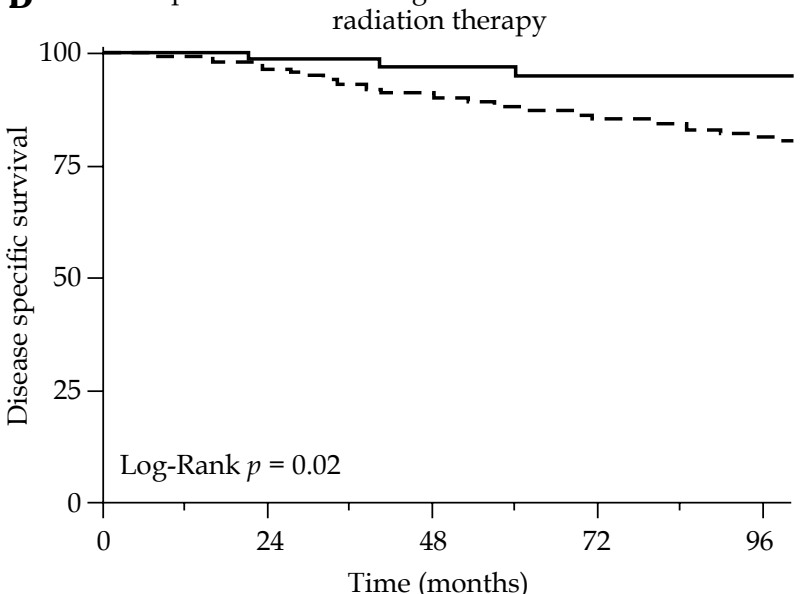

$\begin{array}{lccccc}\text { LSRA } 80 & 64 & 57 & 35 & 20 \\ \text { RT } & 1993 & 1437 & 952 & 579 & 234 \\ & & & & \end{array}$

D Comparison 4: Radiation therapy alone vs. radiation therapy and supplemental laser therapy

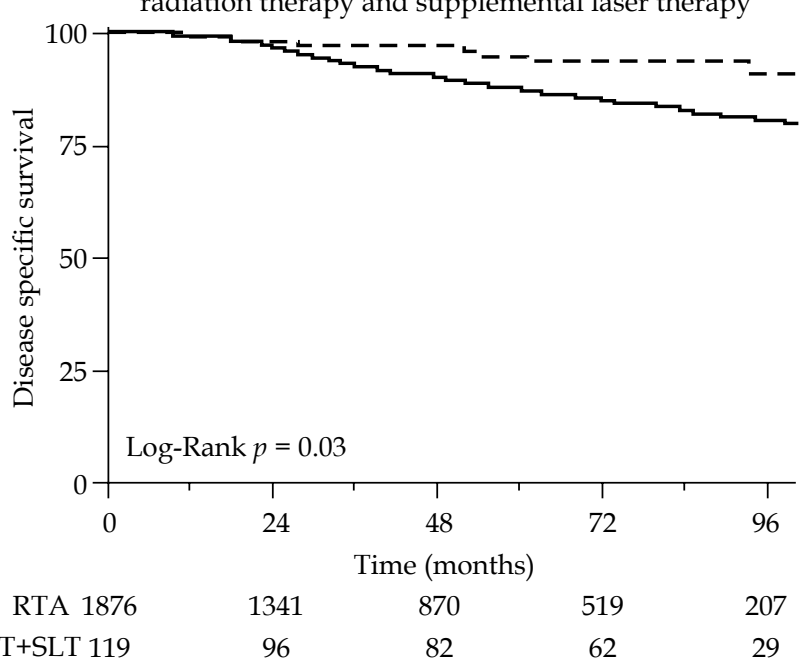

Fig. 5. Disease specific survival according to treatment comparisons

Thus, enucleated patients in the study already had more advanced disease and increased risk of metastasis, and therefore would be at a risk for decreased survival. The 5 -year overall survival of $59 \%$ for enucleation in the current study is lower than that observed in the COMS medium study (82\%) [3], but was similar to that observed in the COMS large study (57\%) [22], providing additional support suggesting that our survival results for enucleation can be explained by a shift in patterns of care toward larger tumors.

One study by Zimmerman and McLean postulated that enucleation might adversely lead to a rapid rise in mortality during the second post-enucleation year. They observed that the fatality rate of patients with posterior uveal melanomas increased from $1 \%$ before enucleation to $8 \%$ during the second year after enucleation. They attribute an increase in metastasis to a shower of emboli released during enucleation, when there is an intraocu- lar pressure spike upon cutting the optic nerve causing dissemination of tumor cells through the vortex veins into systemic circulation [23]. However, concerns about the Zimmerman hypothesis are no longer entertained as more recent evidence suggests that micrometastases occur months before enucleation [24].

Additionally, our results agree with COMS in suggesting that survival outcomes for localized therapy were not inferior compared with enucleation. These findings support the continued use of globe preserving treatments. However, we cannot extrapolate these results to juxtapapillary melanomas or melanoma with extraocular extension, as this information is unavailable in SEER. Ultimately, we believe that the decision between globe preservation treatments and enucleation should be an individualized decision based on patient preferences, and the feasibility of preservation due to vision status, tumor location, and tumor size. 


\section{Local surgical treatment or laser ablation versus radiation for globe preservation}

Our study found increased overall survival for choroidal melanoma patients treated with local resection versus radiotherapy. However, the results were not statistically significant in the multivariate analysis when adjusted for age, race, and stage of tumors. Local resection techniques are technically difficult, and are only utilized in select cases of ciliary or iris tumors or peripheral choroidal melanomas. Two methods of local resection include exoresection, which involves a partial lamellar sclerouvectomy (PLSU) and endoresection, which is done using a vitreous cutter during a pars plana vitrectomy. Similar to our results, Augsburger and Bechrakis found no statistically significant difference in the survival rate of patients treated with local resection versus plaque radiotherapy $[25,26]$. Visual outcomes after PLSU appear to be better than radiation treatment. Bechrakis et al. reported that $61.61 \%$ of eyes harboring a large uveal melanoma retained visual acuity of $>20 / 200$ compared with only $5.6 \%$ of eyes after ${ }^{125} \mathrm{I}$ brachytherapy [16]. In another study of 162 patients with choroidal melanoma treated with local resection, $57 \%$ of patients had $6 / 12$ or better vision, and $93 \%$ of these patients having vision of counting fingers or better [17].

Exoresection complications include risk of extrascleral tumor dissemination or wound leakage, vitreous hemorrhage, and perforation of the retina [27]. A major complication of endoresection is the concern that there may be intraoperative dissemination of tumor cells, which could lead to recurrences. Devitalizing the tumor cells with preoperative irradiation before endoresection may decrease this risk, though no prospective studies addressing this issue exist [28]. A few patients in the SEER data received local tumor excision in addition to radiation, and therefore it is not possible to evaluate survival outcomes after this combined treatment strategy given the small sample size.

Our analysis indicates that survival outcomes are similar in local resection versus radiation. Exoresection is helpful in cases of anteriorly located tumors with ciliary body infiltration. For larger tumors with poor prognosis, local resection may be helpful in decreasing the tumor burden from the eye, lowering the risk for toxic tumor syndrome, which can occur after irradiation as well as provide histopathologic and cytogenetic information about the tumor. When feasible, local resection might be advantageous to primary irradiation in decreasing radiation-induced side effects such as neovascularization and secondary glaucoma, which may lead to better visual outcomes.

\section{External beam versus brachytherapy}

There was no significant difference in the 5-year survival of patients with EBRT versus brachytherapy in our analysis. Though there are no randomized control trials comparing these two treatment modalities for choroidal melanoma, other studies have reported similar results. One previous study of the SEER database also showed no difference in survival between the two treatment modali- ties [10]. The current analysis did not include information on the radio-isotope used for brachytherapy treatment, but ${ }^{125} \mathrm{I}$ is one of the most commonly used isotopes for this purpose in the United States. Alternatively, some centers have used other isotopes such as ${ }^{106} \mathrm{Ru}[29]$ or ${ }^{90} \mathrm{St}$ [29] for ocular brachytherapy.

If survival is equivalent, then the choice of radiation treatment may depend on the differential side effects of the two treatments. Patients treated with radiotherapy develop various side effects such as radiation retinopathy, papillopathy, and cataracts. During external beam radiation, charged particles pass through lids, conjunctiva, cornea, iris, lens, vitreous, and retina before reaching the posterior uveal melanoma. Therefore, this treatment can be more prone to anterior segment and adnexal complications. When compared with charged particle radiotherapy complications, plaque radiotherapy results in more severe radiation retinopathy and optic neuropathy, because plaque irradiation is deposited over the area of the posterior tumor [10]. Eleven percent of patients in one study of $630 \mathrm{pa}-$ tients with macular choroidal melanoma treated with EBRT eventually required enucleation due to side effects and tumor recurrence [30]. A review of 1,019 patients with uveal melanoma treated with plaque brachytherapy showed that $6 \%$ eventually required enucleation of the affected eye [31]. The most common reasons for enucleation in this series included tumor recurrence and neovascular glaucoma.

Visual outcomes vary depending on location and size of the tumors. In a meta-analysis of seven series, $44.6 \%$ of patients treated with radiation alone have maintained better than, or equal to $20 / 200$ vision at an average of 53 months after radiation therapy [32]. Our data suggests that EBRT and brachytherapy provide similar efficacy in tumor treatment. However, our patterns of care data show that plaque radiotherapy in particular, which was used in $53 \%$ of the patients, has become one of the most popular alternatives to enucleation.

\section{Potential benefits of supplemental laser therapy in addition to radiation treatment}

Supplemental laser treatment, such as transpupillary thermotherapy, in addition to radiation may provide better local tumor control in selected patients, but was uncommonly used in our SEER patterns of care analysis. All choroidal melanomas regardless of size have the potential for metastasis, and it is unclear if treating these smaller tumors prevents metastasis. However, in melanomas of other organs, early treatment of the tumors does seem to improve survival [33]. It seems logical that administration of early and effective treatment may increase survival. There are several benefits of transpupillary thermotherapy such as the ability to treat tumors with thickness of more than $5 \mathrm{~mm}$, and the ability to decrease the amount of radiation in the plaque thus limiting the risk for radiation retinopathy. In addition, adjuvant hyperthermia has been shown to make cancers more sensitive to radiation [34]. This may offer improved local control of posterior uveal melanoma tumors, and therefore decreased risk of systemic spread. TTT alone may be insufficient to induce complete tumor necrosis, especially when there is intra 
or episcleral tumor invasion [35]. Transpupillary thermotherapy as an adjunctive treatment has been shown to cause faster tumor regression after brachytherapy in Monosomy 3 choroidal tumors [36]. Several studies have reported a low-rate of recurrence for combination therapy compared to primary plaque radiotherapy or primary TTT. Shield et al. reported a $22 \%$ recurrence rate at 3-year follow-up with sole treatment of TTT versus a $3 \%$ rate of recurrence with combined plaque radiotherapy and TTT [13]. Badiyan et al. reported the results of ${ }^{125} \mathrm{I}$ episcleral plaque brachytherapy with or without TTT in 526 patients with uveal melanoma [15]. In this series, TTT was selectively used in patients at high-risk of recurrence, based on ultrasonic evaluation of plaque tilt. This risk-stratified treatment strategy also resulted in a low local recurrence rate of $3.6 \%$.

The SEER data identified an association of RT+SLT with improved survival from uveal melanoma compared to radiation alone on the univariate analysis with a remarkable absolute difference of $87 \%$ vs. $95 \%$ at 5 -years. However, this association was not significant on the multivariate analysis, and therefore the survival differences could be attributed to treatment selection bias. Indeed, our patterns of care data suggest that supplemental laser therapy was more often used in patients with earlier T-stage and smaller tumors. Therefore, our study could not confirm if TTT or other supplemental laser therapies impact survival. More research is needed to determine whether the possible improved local control associated with supplemental laser therapy in addition to radiation translates into improved survival.

\section{Limitations}

The current retrospective study broadly surveyed the United States SEER database in the modern era to evaluate recent patterns of care, and to report survival outcomes. The retrospective nature of this analysis results in many limitations and significant caution should be applied to the interpretation of the survival comparisons in this study, as selection bias in the choice of treatments may be a confounder in estimating the effect of treatment strategy on patient survival. Most importantly, the results in this study do not supersede the observation in a randomized trial that enucleation and brachytherapy result in similar survival outcomes in uveal melanomas of medium size [21]. Additional limitations of this study include absence of tumor data such as anatomical location, proximity to optic nerve and foveola, largest thickness, pigmentation, and presence of subretinal fluid. The exact tumor size was not known for a substantial proportion of the patients, but the analysis was repeated in the subset of patients with known tumor size to confirm our results. Additionally, the apical height of the tumor, another important prognostic factor, was not analyzed in this population, except as an integrated factor in the T-stage. The radio-isotope used for those patients treated with brachytherapy was unknown in this study. The SEER data does not report any final visual potential after treatment nor is there any data on the toxicity of treatment. In addition, our median follow-up time of 44 months only allows us to reasonably compare survival of these patients at 5 years. In addition, details about radiotherapy treatment protocols are not provided in the SEER database. Our data does not differentiate risk of metastasis and death based on the newest gene expression profiling techniques. Gene expression profiling techniques-based risk assessment may be the most accurate method for predicting survival [37].

\section{Conclusions}

For patients with uveal melanoma treated in the modern era, this retrospective analysis of the SEER database shows that globe preserving treatment is used in a majority of patients with uveal melanoma, and the survival outcomes support its continued use as an alternative to enucleation. External beam radiation therapy and brachytherapy have similar survival outcomes. Prospective trials are necessary to determine the long-term survival of these patients treated with the various strategies. Over the next decade, there is a hope that genetic methods can better identify patients with high-risk for recurrence or metastasis, and so that physicians can administer timely and appropriate treatment to improve these patients' survival as well as achieve preservation of vision.

\section{Acknowledgements}

This work was presented in-part at the ASTRO annual meeting in 2015. The authors would like to thank the National Cancer Institute and the Surveillance, Epidemiology, and End Results Program for their work in creating the SEER database and making it available to researchers.

\section{Disclosure}

Authors report no conflict of interest.

\section{References}

1. Singh AD, Turell ME, Topham AK. Uveal melanoma: Trends in incidence, treatment, and survival. Ophthalmology 2011; 118: 1881-1885.

2. Chattopadhyay C, Kim DW, Gombos DS et al. Uveal melanoma: From diagnosis to treatment and the science in between. Cancer 2016; 122: 2299-2312.

3. Diener-West M, Earle JD, Fine SL et al. Collaborative Ocular Melanoma Study Group, The COMS randomized trial of iodine 125 brachytherapy for choroidal melanoma, III: initial mortality findings. COMS Report No. 18. Arch Ophthalmol 2001; 119: 969-982.

4. Mahendraraj R, Laum K, Lee CS et al. Trends in incidence, survival, and management of uveal melanoma: a population-based study of 7,516 patients from the Surveillance, Epidemiology, and End Results database (1973-2012). Clin Ophthalmol 2016; 2113-2119.

5. The Collaborative Ocular Melanoma Study (COMS) randomized trial of pre-enucleation radiation of large choroidal melanoma III: local complications and observations following enucleation COMS report no. 11. Am J Ophthalmol 1998; 126: 362-372.

6. Andreoli MT, Mieler WF, Leiderman YI. Epidemiological trends in uveal melanoma. Br J Ophthalmol 2015; 99: 1550-1553.

7. Munzenrider JE. Uveal melanomas: Conservation treatment. Hematol Oncol Clin North Am 2001; 15: 389-402. 
8. Wilson MW, Hungerford JL. Comparison of episcleral plaque and proton beam radiation therapy for the treatment of choroidal melanoma. Ophthalmology 1999; 106: 1579-1587.

9. Wackernagel W, Holl E, Tarmann L et al. Local tumour control and eye preservation after gamma-knife radiosurgery of choroidal melanomas. Br J Ophthalmol 2014; 98: 218-223.

10. Abrams MJ, Gagne NL, Melhus CS et al. Brachytherapy vs. external beam radiotherapy for choroidal melanoma: Survival and patterns-of-care analyses. Brachytherapy 2016; 15: 216-223.

11. Oosterhuis JA, Journée-de Korver HG, Kakebeeke-Kemme $\mathrm{HM}$ et al. Transpupillary thermotherapy in choroidal melanomas. Arch Ophthalmol 1995; 113: 315-321.

12. Shields CL, Shields JA, Perez N et al. Primary transpupillary thermotherapy for small choroidal melanoma in 256 consecutive cases: Outcomes and limitations. Ophthalmology 2002; 109: 225-234.

13. Shields CL, Cater J, Shields JA et al. Combined plaque radiotherapy and transpupillary thermotherapy for choroidal melanoma: tumor control and treatment complications in 270 consecutive patients. Arch Ophthalmol 2002; 120: 933-940.

14. Mashayekhi A, Shields CL, Rishi P et al. Shields, Primary Transpupillary Thermotherapy for Choroidal Melanoma in 391 Cases. Ophthalmology 2015; 122: 600-609.

15. Badiyan SN, Rao RC, Apicell AJ et al. Outcomes of iodine-125 plaque brachytherapy for uveal melanoma with intraoperative ultrasonography and supplemental transpupillary thermotherapy. Int J Radiat Oncol Biol Phys 2014; 88: 801-805.

16. Bartlema YM, Oosterhuis JA, Journée-De Korver JG et al. Combined plaque radiotherapy and transpupillary thermotherapy in choroidal melanoma: 5 years' experience. $\mathrm{Br} J$ Ophthalmol 2003; 87: 1370-1373.

17. Damato BE, Paul J, Foulds WS. Predictive factors of visual outcome after local resection of choroidal melanoma. $\mathrm{Br} \mathrm{J} \mathrm{Ophthal-}$ mol 1993; 77: 616-623.

18. Surveillance, Epidemiology, and End Results (SEER) Program; www.seer.cancer.gov.

19. ICD-O-3 Coding Materials, (n.d.); https://seer.cancer.gov/ icd-o-3/ [Accessed: 8 October 2017].

20. Greene F, Balch C, Haller D et al. AJCC Cancer Staging Manual. $6^{\text {th }}$ ed., 2002.

21. Collaborative Ocular Melanoma Study Group. The COMS randomized trial of iodine 125 brachytherapy for choroidal melanoma: V. Twelve-year mortality rates and prognostic factors: COMS report No. 28. Arch Ophthalmol 2006; 124: 1684-1693.

22. The Collaborative Ocular Melanoma Study (COMS) randomized trial of pre-enucleation radiation of large choroidal melanoma II: initial mortality findings. COMS report no. 10. Am J Ophthalmol 1998; 125: 779-796.

23. Zimmerman LE, McLean IW, Foster WD. Does enucleation of the eye containing a malignant melanoma prevent or accelerate the dissemination of tumour cells. $\mathrm{Br} J$ Ophthalmol 1978; 62: 420-425.

24. Singh D, Rennie IG, Kivela T et al. The Zimmerman-McLeanFoster hypothesis: 25 years later. $\mathrm{Br} J$ Ophthalmol 2004; 88: 962-967.

25. Augsburger JJ, Lauritzen K, Gamel JW et al. Matched group study of surgical resection versus cobalt- 60 plaque radiotherapy for primary choroidal or ciliary body melanoma. Ophthalmic Surg 1990; 21: 682-688.

26. Bechrakis NE, Bornfeld N, Zöller I et al. Iodine 125 plaque brachytherapy versus transscleral tumor resection in the treatment of large uveal melanomas. Ophthalmology 2002; 109: 1855-1861.

27. Shields JA, Shields CL, Shah P, et al. Partial lamellar sclerouvectomy for ciliary body and choroidal tumors. Ophthalmology 1991; 98: 971-983.
28. Bechrakis NE, Foerster MH. Neoadjuvant proton beam radiotherapy combined with subsequent endoresection of choroidal melanomas. Int Ophthalmol Clin 2006; 46: 95-107.

29. Naseripour M, Jaberi R, Sedaghat A et al. Ruthenium-106 brachytherapy for thick uveal melanoma: reappraisal of apex and base dose radiation and dose rate. J Contemp Brachytherapy 2016; 8: 66-73.

30. Gündüz K, Shields CL, Shields JA et al. Radiation complications and tumor control after plaque radiotherapy of choroidal melanoma with macular involvement. Am J Ophthalmol 1999; 127: 579-589.

31. Shields CL, Shields JA, Karlsson U et al. Reasons for Enucleation after Plaque Radiotherapy for Posterior Uveal Melanoma. Ophthalmology 1989; 96: 919-924.

32. Finger PT. Radiation therapy for choroidal melanoma. Surv Ophthalmol 1997; 42: 215-232.

33. Berwick M. Why are people still dying from melanoma? Arch Dermatol 1999; 135: 1534-1536.

34. Journee-de Krver H, Schalij-Delfos N, Imhof S. Uveal malignant melanoma: management options - thermotherapy. In: Clinical Ophthalmic Oncology. Singh A, Damato B, Pe'er J (eds.). Saunders Elsevier, Philadelphia 2007; 232-240.

35. Journée-De Korver H, Oosterhuis JG, de Wolff-Rouendaal JA et al. Histopathological findings in human choroidal melanomas after transpupillary thermotherapy. $\mathrm{Br} J$ Ophthalmol 1997; 81: 234-239.

36. Shields CL, Bianciotto C, Rudich D et al. Regression of uveal melanoma after plaque radiotherapy and thermotherapy based on chromosome 3 status. Retina 2008; 28: 1289-1295.

37. Onken MD, Worley LA, Char DH et al. Collaborative Ocular Oncology Group report number 1: prospective validation of a multi-gene prognostic assay in uveal melanoma. Ophthalmology 2012; 119: 1596-1603. 